\title{
Numerical identification of effective multipole moments of polarizable particles
}

\author{
Abdellah Ogbi*, Laurent Nicolas*, Ronan Perrussel ${ }^{\dagger}$, Sheppard J. Salon ${ }^{\ddagger}$, and Damien Voyer* \\ *Laboratoire Ampère CNRS UMR5005, Université de Lyon, École Centrale de Lyon, Écully, France \\ ${ }^{\dagger}$ LAPLACE CNRS UMR5213, Université de Toulouse, INPT-ENSEEIHT, Toulouse, France \\ ${ }^{\ddagger}$ Electrical, Computer, and Systems Engineering Department, Rensselaer Polytechnic Institute, Troy, USA \\ mail to: abdellah.ogbi@ec-lyon.fr
}

\begin{abstract}
In electromechanics of particles, the effective moment method relies on the knowledge of the induced multipole moments. For arbitrary shaped particles, only linear multipole moments are usually considered in the literature. On cylindrically symmetric particles, we show that the neglected multipole moments can be computed, and that they should be taken into consideration.
\end{abstract}

Index Terms-Electromechanical effects, multipole moments, polarization.

\section{INTRODUCTION}

W HEN a dielectric particle is subjected to an electrostatic field, interfacial polarization mechanisms cause a charge accumulation on the surface between the particle and the medium in which it is suspended [1], [2]. Usually this polarization gives rise to an induced dipole that will interact with the external field to create forces and torques [1], [3]. This interaction is exploited in the dielectrophoretic phenomena such as electrorotation and dielectrophoresis for the manipulation and the characterization of biological particles [1], [3].

In the conventional dielectrophoresis theory, it is usually assumed that only a dipole is induced [4], [5]. Although this approximation seems to be adequate in many circumstances, exceptions exist and higher-order multipole corrections are required to predict the particle behavior. In particular, these higher-order corrections can influence forces and torques exerted on cells in planar quadrupolar traps and multilayer structures when the particle is at a zero field, or when the particle size becomes comparable to the dimensions of the electrode structure used to generate the electric field [6]-[8].

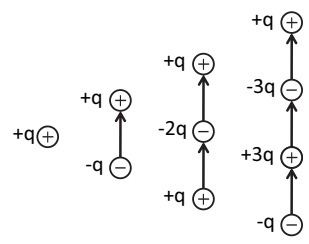

Fig. 1. Stratton's representation of the first four linear multipoles.

The effective moment method developed by Jones and Washizu, use higher-order multipole moments to provide a more accurate estimation of the force and the torque exerted upon the manipulated particle [9]-[11]. In this method, the particle under consideration is substituted by a set of multipoles (dipole, quadrupole, octupole,...) producing the same field distortion caused by the presence of the particle; the force exerted on multipoles should be equal to the force exerted on the particle by the external field [3], [12].

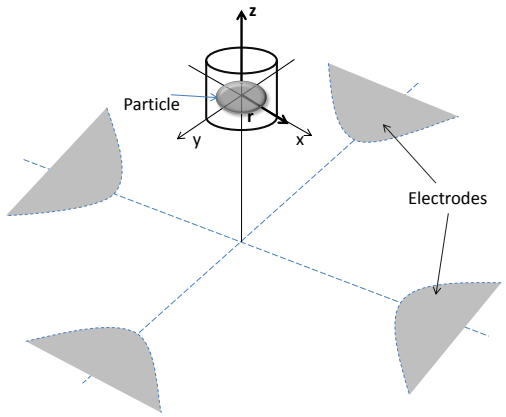

Fig. 2. Example of a 3D system of electrodes and a particle.

The simplest multipole model considers only the linear multipoles. Green and Jones use this model when determining the dipole moment and higher-order moments [13]. The linear multipoles can be represented by an arrangement of equally spaced positive and negative charge centers along the axis of the dipole (Fig. 1). However, this linear multipole model cannot be used when the external field is not aligned with the symmetry axis of a cylindrically symmetric particle. It is unfortunately the most frequent situation in biological dielectrophoretic applications where, for instance, the radial component of the field is higher than the $z$-component (Fig. 2).

Here, we also take into account the neglected multipole moments to identify an equivalent model of the polarized particle (Fig. 3) from the induced potential. The multipole expansion is first recalled. On cylindrically symmetric particles, a procedure is then proposed to compute all the multipole moments. Numerical results show the relevance of the approach.

\section{MODEL AND METHOD}

\section{A. Induced potential}

Consider a particle immersed in a homogeneous medium and exposed to an electric field generated by an external system. The particle and the surrounding medium are assumed to have linear dielectric properties. The electrostatic potential in the system containing the different materials, is obtained by solving a Laplace type equation. 


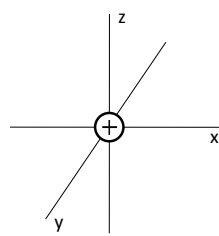

$(0,0)$

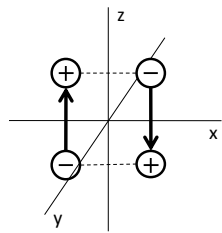

$(2,1)$

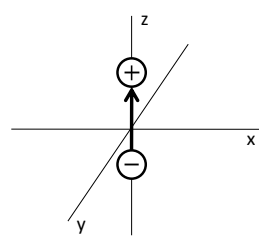

$(1,0)$

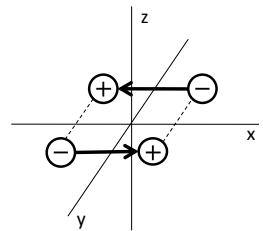

$(2,2)$

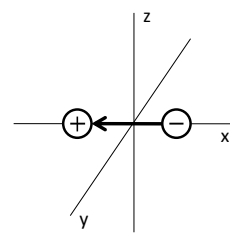

$(\mathbf{1}, \mathbf{1})$

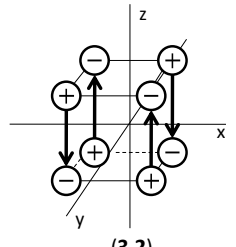

$(3,2)$
Fig. 3. A 3D representation of general multipoles with the corresponding orders $(n, m)$.

The total electric potential $U_{\text {total }}$ outside the particle is the sum of both applied potential $U_{\text {ext }}$ and induced potential $U_{\text {ind }}$ caused by the polarization of the particle, i.e.

$$
\begin{aligned}
U_{\text {total }} & =U_{\text {ext }}+U_{\text {ind }} \\
& =\sum_{n, m} A_{n, m} r^{n} \mathcal{Y}_{n}^{m}(\theta, \varphi)+\sum_{n, m} \frac{B_{n, m}}{r^{n+1}} \mathcal{Y}_{n}^{m}(\theta, \varphi),
\end{aligned}
$$

where $\mathcal{Y}_{n}^{m}$ are the complex spherical harmonics, and $(r, \theta, \varphi)$ a spherical coordinate system. The magnitudes $A_{n, m}$ and $B_{n, m}$ can be determined numerically or analytically using proper boundary conditions.

The multipole polarization coefficients $p_{n}^{m}$ are related to the induced potential constants $B_{n, m}$ by

$$
p_{n}^{m}=\mathcal{N}_{n}^{m} B_{n, m}, \text { with } \mathcal{N}_{n}^{m}=\frac{(2 n+1)}{4 \pi} \sqrt{\frac{(n-m) !}{(n+m) !}} .
$$

The linear multipoles correspond to the case $m=0$ in the expression (2). For a spherical particle with radius $a$ and permittivity $\varepsilon_{2}$ suspended in a medium with permittivity $\varepsilon_{1}$, the $B_{n, m}$ can be expressed in terms of the $A_{n, m}$ [3]:

$$
B_{n, m}=\frac{K_{n}}{(2 n+1)} a^{2 n+1} A_{n, m},
$$

where

$$
K_{n}=\frac{n(2 n+1)\left(\varepsilon_{1}-\varepsilon_{2}\right)}{(n+1) \varepsilon_{2}+n \varepsilon_{1}}
$$

is the generalized Clausius-Mossotti factor.

\section{B. Calculation of the effective multipole moments}

The constants $A_{n, m}$ extracted from the calculation of the potential in the system without the particle are used as source terms for a local problem including the particle. Then, based on the spherical harmonics representation for the induced potential, we identify the effective moments.

Using the orthogonality property of the spherical harmonics the individual moments are determined according to

$$
p_{n}^{m}=\mathcal{N}_{n}^{m} R^{n+1} \int_{S} \mathcal{Y}_{n}^{m}(\theta, \varphi) U_{\text {ind }}(R, \theta, \varphi) d s,
$$

where $S$ is a spherical surface centered on the particle with a radius $R$ larger than the largest particle dimension (Fig. 4).

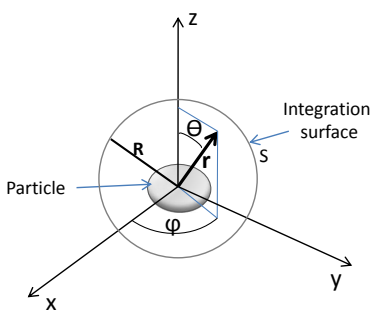

Fig. 4. Integration surface around the particle.

\section{Solution of the problem}

The particle of interest in our problem is cylindrically symmetric with permittivity $\varepsilon_{2}$, immersed in a containing medium with permittivity $\varepsilon_{1}$. The particle is assumed to be sufficiently small to consider the external electric field constant in its neighborhood. Consequently, the 3D problem is transformed into two axisymmetric problems (Fig. 5). The

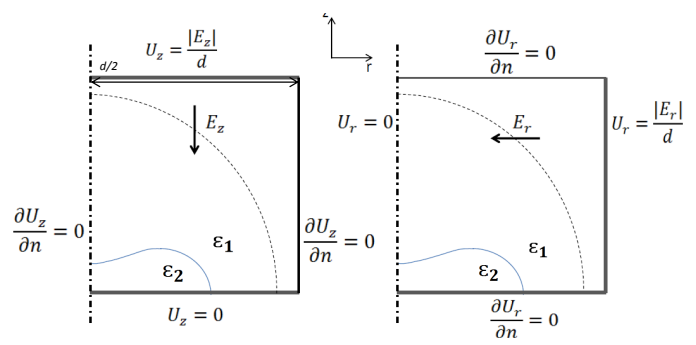

Fig. 5. Boundary conditions for the radial and z-axis potential calculations.

electric potential in the whole space is obtained using both solutions:

$$
U(r, z, \theta)=U_{z}+U_{r} \cos (\varphi)
$$

Two linear problems for $U_{z}$ and $U_{r}$ are then required to be solved in the computational domain $\Omega$ :

$$
\int_{\Omega} r\left(\frac{\partial U_{r}}{\partial r} \frac{\partial v}{\partial r}+\frac{\partial U_{r}}{\partial z} \frac{\partial v}{\partial z}\right) d r d z+\int_{\Omega} \frac{U_{r}}{r} v d r d z=0
$$

and

$$
\int_{\Omega} r\left(\frac{\partial U_{z}}{\partial r} \frac{\partial v}{\partial r}+\frac{\partial U_{z}}{\partial z} \frac{\partial v}{\partial z}\right) d r d z=0
$$

\section{NUMERICAL RESULTS}

The numerical solution of problems (7) and (8) is performed by using the finite element method and the implementation is based on the get fem++ finite element library [14] (Fig. 6).
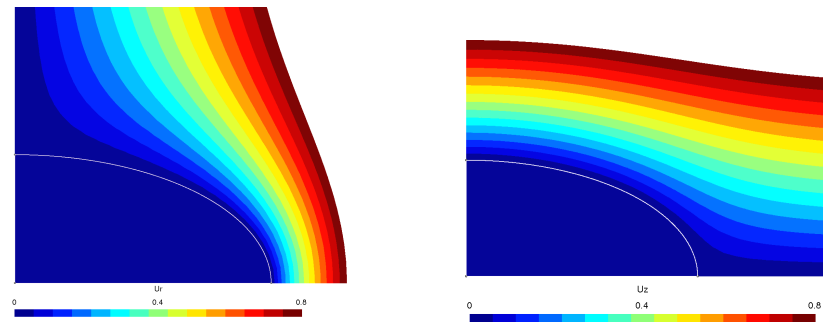

Fig. 6. Details of the solutions $U_{r}$ (left) and $U_{z}$ (right). The white line is the boundary of the particle. $\varepsilon_{2} / \varepsilon_{1}=10^{4}$. 

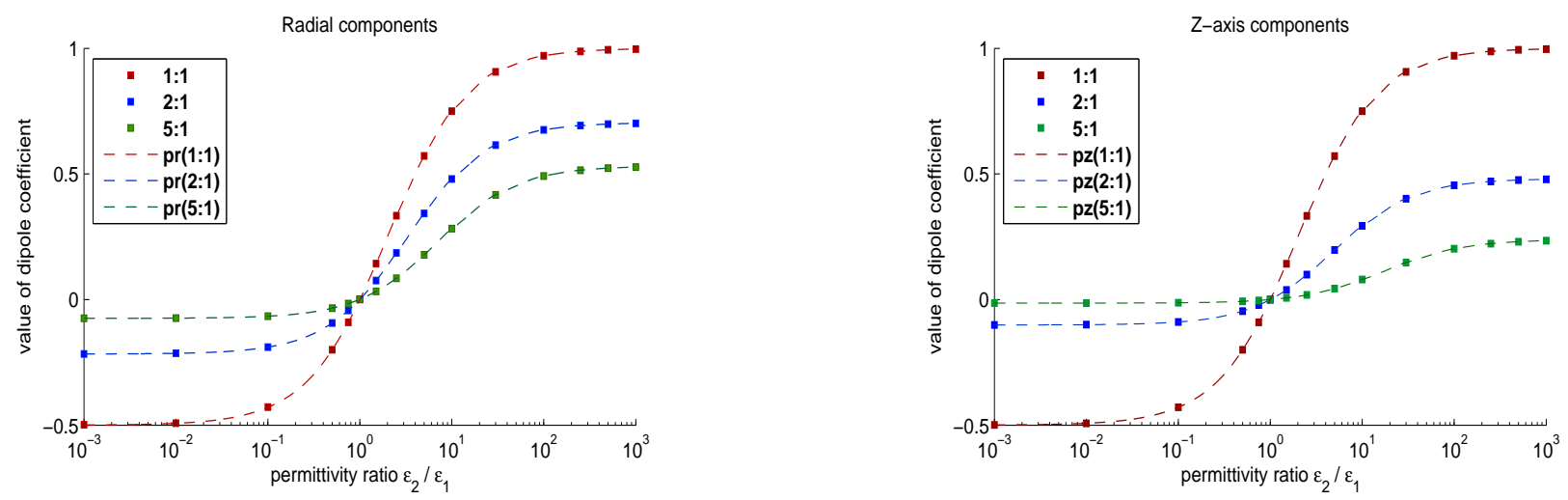

Fig. 7. Radial and z-axis components of the dipole moment for a spheroidal particle. The numerical results are plotted as symbols, while the corresponding analytical values $p_{r}$ and $p_{z}$, given by (9) and (10), are plotted as dotted lines.

The geometries and the adaptive mesh of the computational domain, were built using the three-dimensional finite element mesh generator Gmsh, and the numerical integration required in (5), was carried out using Gaussian quadrature. The method described in the previous section enables to handle nonspherical particles, for which there is no analytical method for identifying the higher-order moments. A particular shape frequently invoked in biological cells modeling [15], is the spheroidal shape. By changing the eccentricity ratio (Fig. 8), the shape dependence of the particle polarization can be identified (Fig. 9).

\section{A. Dipole moment}

An analytical representation of the dipole moment for the ellipsoidal shape exists. In particular for a spheroid with eccentricity ratio $\alpha=c / a$ (Fig. 8), the dipole polarization factors are given by:

$$
\begin{aligned}
& p_{r}=\frac{c a^{2}}{3} \frac{\left(\varepsilon_{2}-\varepsilon_{1}\right)}{\left[\varepsilon_{2}+L_{r}\left(\varepsilon_{1}-\varepsilon_{2}\right)\right]} \\
& p_{z}=\frac{c a^{2}}{3} \frac{\left(\varepsilon_{2}-\varepsilon_{1}\right)}{\left[\varepsilon_{2}+L_{z}\left(\varepsilon_{1}-\varepsilon_{2}\right)\right]}
\end{aligned}
$$

where the magnitudes $L_{r}$ and $L_{z}$ are the depolarization factors, given for the case of an oblate spheroid by [16]:

$$
\begin{aligned}
L_{r} & =\frac{1}{2\left(1-\alpha^{-2}\right)}-\frac{\alpha^{-2}}{4\left(1-\alpha^{-2}\right)^{3 / 2}} \ln \frac{1+\sqrt{1-\alpha^{-2}}}{1-\sqrt{1-\alpha^{-2}}}, \\
L_{z} & =\frac{\alpha^{-2}}{1-\alpha^{-2}}+\frac{\alpha^{-2}}{2\left(1-\alpha^{-2}\right)^{3 / 2}} \ln \frac{1+\sqrt{1-\alpha^{-2}}}{1-\sqrt{1-\alpha^{-2}}} .
\end{aligned}
$$

For the spherical case, the depolarization factors verify $L_{r}=L_{z}=1 / 3$, and the polarization coefficients are given by Clausius-Mossotti factor $K_{1}$. In Fig. 7, these analytical values are compared with the computed values of the dipole moment and it shows a good agreement.

\section{B. Higher-order moments}

The higher-order moments are obtained only numerically using the proposed approach. The first case considered is a homogeneous spherical particle $(\alpha=1)$. In this case, only the dipole moment is non-zero and matches well with the
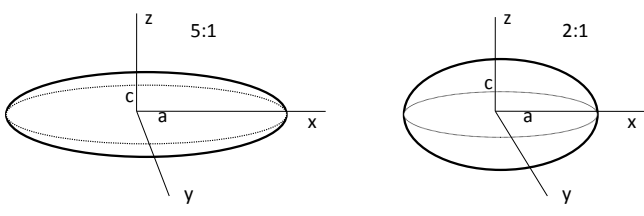

Fig. 8. Oblate spheroids with eccentricities 5:1 and 2:1.

analytical values given by the Clausius-Mossotti factor $K_{1}$. As it was expected from the isotropy of the sphere, note that the radial and the $\mathrm{z}$-axis dipole moments have the same magnitude (see upper row in Fig. 9).

For lower eccentricities i.e $\alpha<1$ (oblate spheroids), the numerical results in Fig. 9 (middle and lower rows) indicate that the dipole component decreases when $\alpha$ decreases, particularly in the $z$-direction. This behavior is also predictable with the analytical description of the dipole moments with the depolarization factors. Besides, the magnitudes of the identified higher-order components, become significant as $\alpha$ decreases (with $\alpha<1$ ), in particular, the octupole moments has important magnitudes in both the radial and the $\mathrm{z}$-axis directions, with larger values when the particle permittivity is greater than the surrounding medium permittivity.

\section{CONCLUSION}

In this paper, a complete multipole model was exploited in a numerical approach for the identification of the effective multipole moments. This complete multipole model enables to identify multipole components (terms) that are displaced from the central axis $(m \neq 0)$. Only such terms can describe the observed interaction of a particle placed in an external field that is not aligned with its central axis, such as in the center of the quadrupolar electrode structure [7]. The next step in this work is to extend this approach to layered particles model invoked in complex biological cells modeling.

\section{REFERENCES}

[1] H. A. Pohl and J. S. Crane, "Dielectrophoresis of cells." Biophys J, vol. 11 , no. 9 , pp. 711-27, 1971 


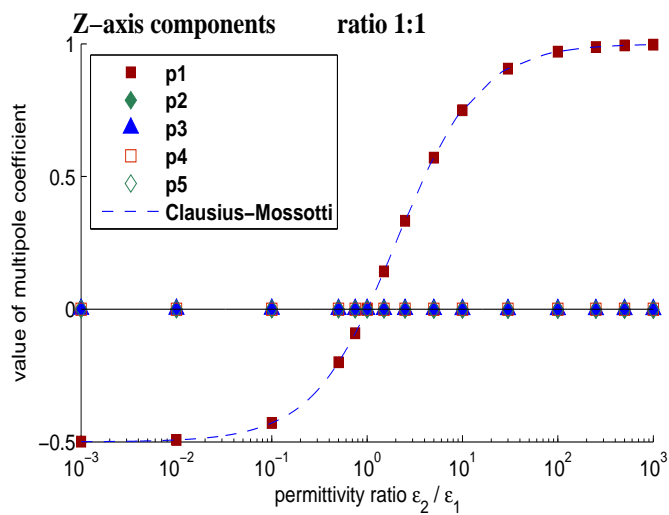

ratio 2:1

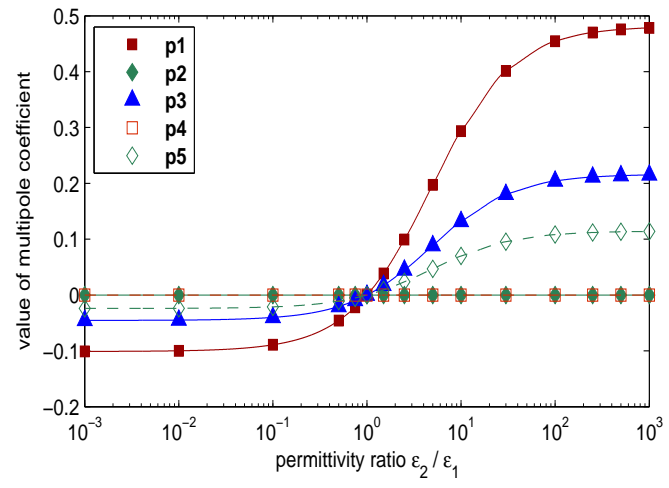

ratio 5:1

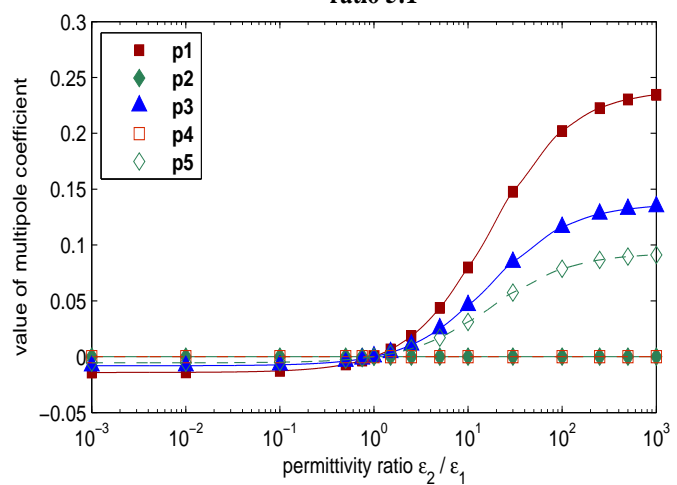

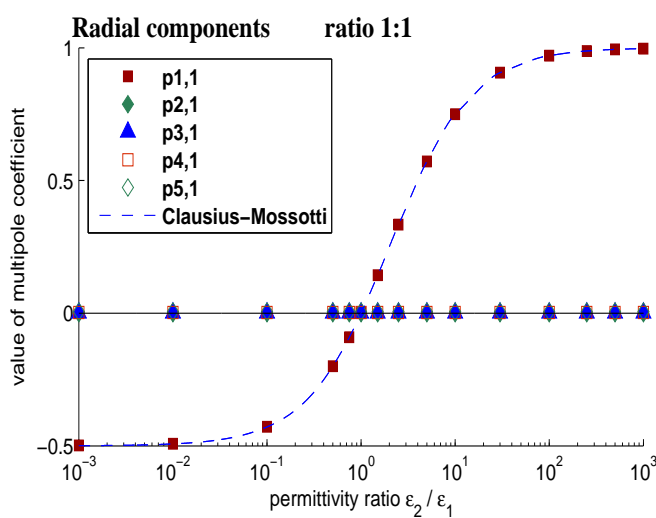

ratio 2:1

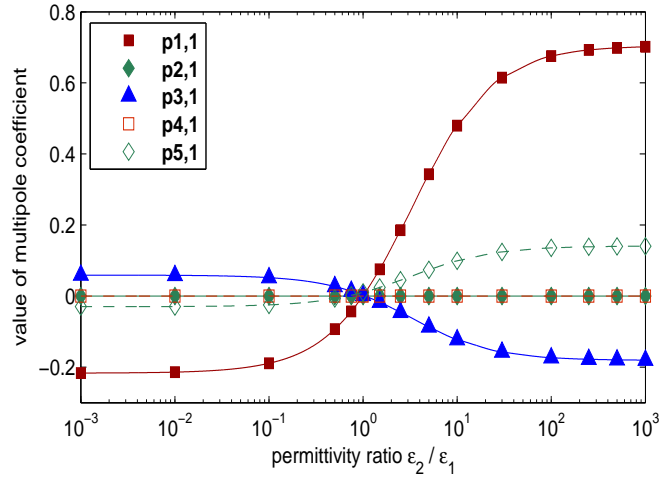

ratio 5:1

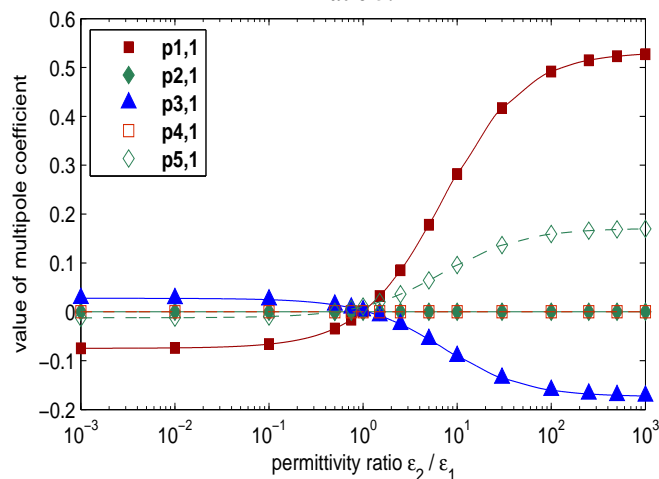

Fig. 9. Numerical results following the radial axis (column left) and the z-axis (column right), for spheroidal (oblate) particles of different aspect ratios (eccentricity) $c / a$. The five first orders are plotted as symbols against the ratio of particle permittivity to fluid medium permittivity $\varepsilon_{2} / \varepsilon_{1}$.

[2] H. Morgan and N. Green, AC electrokinetics: colloids and nanoparticles, ser. Microtechnologies and microsystems series. Philadelphia, PA, USA: Research Studies Press, 2003, no. 2.

[3] T. B. Jones, Electromechanics of particles. Cambridge Univ Press, 1995.

[4] J. Cao, P. Cheng, and F. Hong, "A numerical analysis of forces imposed on particles in conventional dielectrophoresis in microchannels with interdigitated electrodes," Journal of Electrostatics, vol. 66, no. 11-12, pp. 620-626, 2008.

[5] U. Zimmermann and G. A. Neil, Electromanipulation of Cells, 1st ed. CRC-Press, Apr. 1996.

[6] W. Masao, T. B. Jones, and K. V. I. S. Kaler, "Higher-order dielectrophoretic effects: levitation at a field null," Biochimica et Biophysica Acta (BBA) - General Subjects, vol. 1158, no. 1, pp. 40-46, 1993.

[7] T. Schnelle, T. Müller, S. Fiedler, and G. Fuhr, "The influence of higher moments on particle behaviour in dielectrophoretic field cages," Journal of Electrostatics, vol. 46, no. 1, pp. 13-28, Mar. 1999.

[8] T. Schnelle, T. Muller, C. Reichle, and G. Fuhr, "Combined dielectrophoretic field cages and laser tweezers for electrorotation," Applied Physics B Lasers and Optics, vol. 70, no. 2, pp. 267-274, 2000.

[9] T. B. Jones and M. Washizu, "Multipolar dielectrophoretic and electrorotation theory," Journal of Electrostatics, vol. 37, no. 1-2, pp. 121-134,
May 1996.

[10] M. Washizu and T. Jones, "Multipolar dielectrophoretic force calculation," Journal of Electrostatics, vol. 33, no. 2, pp. 187-198, 1994.

[11] M. Washizu, "Equivalent multipole-moment theory for dielectrophoresis and electrorotation in electromagnetic field," Journal of Electrostatics, vol. 62, no. 1, pp. 15 - 33, 2004.

[12] X. Wang, X.-B. Wang, and P. R. Gascoyne, "General expressions for dielectrophoretic force and electrorotational torque derived using the Maxwell stress tensor method," Journal of Electrostatics, vol. 39, no. 4, pp. 277-295, 1997.

[13] N. G. Green and T. B. Jones, "Numerical determination of the effective moments of non-spherical particles," Journal of Physics D: Applied Physics, vol. 40, no. 1, pp. 78-85, 2007.

[14] Y. Renard and J. Pommier, "getfem++ finite element library," http:// download.gna.org/getfem/html/homepage.

[15] L. Gao, J. Huang, and K. Yu, "Theory of ac electrokinetic behavior of spheroidal cell suspensions with an intrinsic dispersion," Physical Review E, vol. 67, no. 2, pp. 1-10, Feb. 2003.

[16] N. Rivette, "A note on the electrostatic force and torque acting on an isolated body in an electric field," Chemical Engineering Science, vol. 51, no. 23, pp. 5205-5211, 1996. 